\title{
Efficient Generation of Inflow Conditions for Large Eddy Simulation of Street-Scale Flows
}

\author{
Zheng-Tong Xie and Ian P Castro \\ School of Engineering Sciences, University of Southampton, SO17 1BJ, UK
}

\begin{abstract}
Using a numerical weather forecasting code to provide the dynamic large-scale inlet boundary conditions for the computation of small-scale urban canopy flows requires a continuous specification of appropriate inlet turbulence. For such computations to be practical, a very efficient method of generating such turbulence is needed.

Correlation functions of typical turbulent shear flows have forms not too dissimilar to decaying exponentials. A digital-filter-based generation of turbulent inflow conditions exploiting this fact is presented as a suitable technique for LES computation of spatially developing flows. The artificially generated turbulent inflows satisfy the prescribed integral length scales and Reynolds-stress-tensor. The method is much more efficient than, for example, Klein's or Kempf et al.'s (2005) methods because at every time step only one set of two-dimensional (rather than three-dimensional) random data is filtered to generate a set of two-dimensional data with the appropriate spatial correlations. These data are correlated with the data from the previous time step by using an exponential function based on two weight factors. The method is validated by simulating plane channel flows with smooth walls and flows over arrays of staggered cubes (a generic urban-type flow). Mean velocities, the Reynolds-stress-tensor and spectra are all shown to be comparable with those obtained using classical inlet-outlet periodic boundary conditions. Confidence has been gained in using this method to couple weather scale flows and street scale computations.
\end{abstract}

Keywords: weather scale, urban CFD, coupling, bluff bodies, channel flow

\section{Introduction}

An understanding of the mechanisms by which the urban boundary layer, the rural boundary layer, the city scale flow (Britter \& Hanna, 2003), the regional weather and the general circulation of the atmosphere, are coupled aerodynamically and thermodynamically is very important but still in its infancy. For instance, the temperature in cities has been found to be up to ten $\operatorname{deg} \mathrm{C}$ warmer than the surrounding rural areas, and to cause large increases in rainfall amounts downwind; however, there are situations in which urban aerosol suppresses precipitation (Collier, 2006). In most general circulation models (GCM) or operational regional weather models like the UK Meteorological Office's Unified Model (UM) a single numerical grid has an axial length of no less than about one kilometer. Increasing the resolution of such models to, say, one meter is impractical and will remain so for the foreseeable future, so to investigate the detailed micro-meteorology within an urban area another approach is needed. Our eventual objective is therefore to develop tools for providing spatial boundary conditions derived from large-scale computations (e.g. from the UM) to 'more classical' Large Eddy Simulations (LES) of the localised, small-

(c) 2008 Kluwer Academic Publishers. Printed in the Netherlands. 
scale flows within urban canopy regions. Such a localised domain may in fact be smaller than the typical grid size of a 'weather' code like the UM. Quite apart from the problems posed by the unsteady and turbulent boundary conditions, typical urban areas comprising buildings, streets, parks, etc. present a considerable challenge for current engineering-type CFD tools. For adequate prediction of processes like the unsteady dispersion of pollutants it is arguably necessary to use genuinely unsteady methods like LES but this seriously exacerbates the difficulties posed by having to supply appropriate boundary conditions. One requires a very efficient method to insert appropriate small-scale turbulence at the upstream boundary at each time step, recognising that the flow is almost never close to being spatially periodic. The much larger scale fluctuations can then be provided by output from the large-scale model (e.g. the UM).

The most direct method would be to simulate a laminar inflow and allow it to develop spatially over a suitably long domain, i.e. over a hundred times the thickness of the eventual boundary layer depth of interest. However, even this 'simple' method would present difficulties associated with, amongst other things, ensuring the correct surface condition. It would in any case be extremely expensive computationally. Moreover, for what we might call 'urban CFD', only a part of the urban area might be of interest and the upstream geometry may be hard to resolve properly. This method is thus rarely used and is not really applicable for urban CFD.

Alternatively, a time-evolving LES or direct numerical simulation (DNS) with a periodic (Garcia et al., 2004; Keating et al., 2004) or a 'modified periodic' (Lund et al., 1998) inlet-outlet boundary condition can be used to generate appropriate turbulent flow. The latter authors used a sort of 'precursor simulation', in which the velocity field at an appropriate downstream station is stored and imposed in suitably re-scaled form as inflow data for the primary computation. The precursor simulation generates a realistic turbulent flow field. However, it is also expensive and has limitations, being applicable for example to simple geometries only. It is difficult to see how such a method could be used for a genuine urban-type situation.

Procedures based on proper orthogonal decomposition (POD) (Johansson \& Andersson, 2004; Druault et al., 2004; Perret et al., 2006) are probably less expensive for the generation of inflow data than the above methods, provided there are appropriate DNS/LES (Johansson \& Andersson, 2004) or experimental datasets (Druault et al., 2004; Perret et al., 2006) available for processing to obtain the most energetic modes, with an appropriate set of time dependent projection coefficients which provide the phase information. The reconstructed inflow data for later LES/DNS are 'almost realistic'. However, the experimental databases suffer from either low spatial resolution, common with measurements from hot-wire or Laser Doppler Anemometry (Druault et al., 2004), or low temporal resolution, as from measurements using Particle Image Velocimetry (Perret et al., 2006). Special treatment must then be applied to resolve the low resolution issues. Consequently, such approaches are likely to be suitable only for very particular cases.

Synthetic turbulence generation is another option. This is of particular interest when only limited turbulence statistics data are available for the procedure. Hanna et al.(2002) 
generated one-dimensional time series of inflow data based on an exponential correlation function to simulate flows over an array of cubes using LES. The time series were tailored to provide the required time scale and turbulence intensities and the subsequent LES was able to reproduce the main characteristics of the measurements. The merit of the method is its very high efficiency, but because no spatial correlation was imposed at inlet its accuracy is seriously limited.

Inverse Fourier transforms applied to prescribed spectra are able to generate artificial turbulent inflows which preserve both the given spatial and temporal correlations (Kondo et al., 1997; Lee et al., 1992). A long distance is required to recover 'realistic' turbulence. In a channel flow simulation, for example, twenty boundary layer thicknesses were required to recover the correct skin friction (Le \& Moin et al., 1994). Because it might be fairly expensive to obtain detailed spectra, these methods were not considered for the simulations of street-scale flows .

Batten et al.(2004) reconstructed an inflow turbulent fluctuation field by summing a set of sines and cosines with carefully designed random phases and amplitudes, preserving a given set of space/time correlations and a set of second order moments. The procedure is easy to code and the efficiency is high. However, Keating et al.(2004) used this procedure to generate inflow data for a plane channel flow and found that the redevelopment was slow - after a distance of twenty channel half-heights the turbulence in the core was clearly not yet fully developed.

Klein et al.(2003) developed a technique generating artificial velocities as inflow data for jet flows, which reproduced first and second order one-point statistics as well as locally given correlations. The technique was based on the knowledge that for late-stage homogeneous turbulence the correlation function takes a Gaussian form. A three-dimensional digital filter was therefore used to obtain two-dimensional slices of data, arranged in the axial direction and with appropriate spatial correlations, from a set of three-dimensional random data, i.e. a pile of slices of random data. The temporal correlations were also preserved by shifting all the slices of random data by one 'step' at the next time step, discarding the slice at the beginning of the pile of slices and adding a new slice of random data at the other end. Kempf et al.(2005) demonstrated that a procedure solving an appropriate diffusion equation for a set of three-dimensional random data was equivalent to applying a Gaussian function based filter for the same random data. Since the diffusion was applied in physical space, we speculate that the phases of the synthetically correlated inlet data must also have been incorporated in the inflow data, at least to some extent and albeit implicitly. This could be another significant advantage of these procedures.

di Mare et al.(2006) constructed a digital filter based on a relatively arbitrary correlation function, which could be simply assumed in a Gaussian shape, an exponential shape, or be obtained from experiments or precursor numerical simulations. This is a more general approach in terms of using the correlation functions, which is based on solving an array of equations of the unknown filter coefficients and the correlation coefficients. However, as stressed in the paper (di Mare et al., 2006) the equations are incompatible and the solution is not unique. Most important, there is no guarantee that the equations has a solution for 
any given correlation coefficients. To overcome these, a target - minimum residuals of the equations is sought. This approach was found able to reproduce locally defined, spatial and temporal correlation functions.

Based on the three-dimensional digital filter approach, Veloudis et al. (2007) extensively investigated the effect of using spatially varying turbulence scales as inputs for the generation of inflow conditions (also as mentioned in the above, di Mare et al.used a more general approach to investigate this). In the wall normal direction, the turbulence scale profiles for a channel flow were digitised into several zones, within which the turbulence scales were constants. Using spatially varying, rather than constant scales at the inlet led to better predictions of the test case, on the evidence of the mean and, particularly, the turbulence profiles. They also developed a method to improve the efficiency of the three-dimensional digital filter approach by using the Fast Fourier Transform (FFT) for the filtering process.

Sandham et al.(2003) developed an inflow procedure which introduced specific innerand outer-layer disturbances with associated phase information, which only required a short distance from the inflow to develop the turbulence fully. However, this method aims to model the streaks in the inner layer and consequently it is only suitable for high-resolution LES, i.e. with typically fifteen points in the viscous sublayer. Based on the view that turbulence comprises a superposition of coherent structures or turbulent spots, Jarrin et al.(2006) proposed a synthetic eddy-method for inflow data. This method was very efficient. However the arbitrariness of choosing the location and the size of an individual eddy made it complicated to use.

Because of the features of urban boundary layer flows - high Reynolds number, fully developed turbulence and driven by weather scale motions - a particularly efficient inflow procedure is needed. We resorted to a digital-filter based method and in $\S 3$ we describe a technique based on exponential correlation functions for spatially developing LES. The method allows spatially varying turbulence scales on non-uniform grids to be imposed at the inlet. It was developed independently of the work of di Mare et al. (2006) and Veloudis et al. (2007), whose method is similar in some respects, and has already been used elsewhere (Xie \& Castro, 2005; Xie \& Castro, 2006b). This paper describes the method in detail for the first time (in $\S 3$ ); it is shown to be very much more efficient than its nearest equivalents above - those of Klein et al.(2003) and Veloudis et al. (2007) - and also inherently more suitable for coupling to a large-scale weather code. It is also more accurate than methods which don't allow for spatial variation in the prescribed length scales. The method has been validated by applying it first to an LES simulation of a plane channel flow, as described in $\S 4$. Secondly, $\S 5$ presents results of a simulation of the flow over a group of staggered cubes. The results are compared with measurements and previous DNS computations and with those obtained by using periodic inlet-outlet conditions. 


\section{Governing Equations}

To ensure a largely self-contained paper, a brief description of the governing equations is given here. More details can be found in Xie \& Castro (2006a), hereafter denoted by XC.

The filtered continuity and Navier-Stokes equations are written as follows,

$$
\begin{aligned}
& \frac{\partial u_{i}}{\partial x_{i}}=0 \\
& \frac{\partial u_{i}}{\partial t}+\frac{\partial u_{i} u_{j}}{\partial x_{j}}=-\frac{1}{\rho}\left(\frac{\partial p}{\partial x_{i}}+\delta_{i 1} \frac{\partial\langle P\rangle}{\partial x_{1}}\right)+\frac{\partial}{\partial x_{j}}\left(\tau_{i j}+\nu \frac{\partial u_{i}}{\partial x_{j}}\right) .
\end{aligned}
$$

The dynamical quantities, $u_{i}, p$ are resolved-scale (filtered) velocity and pressure respectively and $\tau_{i j}$ is the subgrid-scale (SGS) Reynolds stress. $\delta_{i 1}$ is the Kronecker-delta and $\nu$ is the kinematic viscosity. $\partial\langle P\rangle / \partial x_{1}$ is the driving force, a constant streamwise pressure gradient which exists only when periodic inlet-outlet boundary conditions are applied but otherwise vanishes. The Smagorinsky SGS model was used with $C_{s}=0.1$. In the near-wall region, the Lilly damping function was also applied. Note that the Smagorinsky model is widely used by researchers to simulate the kind of flow of most concern to us - rough-wall flows - with considerable success (Hanna et al., 2002; Stoesser et al., 2003; Xie \& Castro, 2006a).

The wall model is generally an important issue for LES, and is no less important than the SGS model if the computational cost is to be minimised. For cases where the fine eddies in the vicinity of the wall are important, it is recommended that $\mathscr{N}_{1}^{+}$is of order unity $\left(\mathscr{N}_{1}^{+}\right.$is the distance in wall units between the centroid of the first cell and the wall assuming the $\mathscr{N}$ coordinate is normal to the wall). Note, however, that for a complex geometry, where separation and attachment processes occur, it is impossible to satisfy this criteria everywhere. We argue that, unlike the situation for smooth-wall flows, it is in fact not necessary, at least for obtaining overall surface drag and the turbulent motions at the scale of the roughness elements (buildings), which turn out to be dominant (see XC).

The local wall shear stress is then obtained from the laminar stress-strain relationships:

$$
u^{+}=\frac{u}{\hat{u}_{\tau}}, \mathscr{N}^{+}=\frac{\rho \hat{u}_{\tau} \mathscr{N}}{\mu}, \mathscr{N}^{+}=u^{+},
$$

where $\rho \hat{u}_{\tau}^{2}$ is the local wall shear stress. However, if the near-wall mesh is not fine enough to resolve the viscous sublayer, for simplicity it is assumed that the centroid of the cell next to the wall falls within the logarithmic region of the boundary layer:

$$
\frac{u}{\hat{u}_{\tau}}=\frac{1}{\kappa} \ln E\left(\frac{\rho \hat{u}_{\tau} \mathscr{N}}{\mu}\right)
$$

where $\kappa$ is the von Karman constant and $E$ is an empirical constant. The log-law is employed when $\mathscr{N}^{+}>11.2$. Again, note that for very rough-wall flows there are prob- 
ably very few regions on the surface of the roughness elements where log-law conditions genuinely occur in practice; however, we have shown earlier that for this type of flow the precise surface condition is unimportant for capturing the element-scale flows and surface drag (see XC).

The entire LES model was implemented in the code described in XC. Crucially, the discretisation for all terms in Eq. (1) was second order accurate in both space and time lower-order schemes were found not to be adequate but, equally, for the kind of problems addressed here it is not necessary to use schemes that are of even higher order. Inlet boundary conditions were set using a User-Defined-Function, embodying the technique described in the following section.

\section{Generation of Inflow Data}

The exponential velocity correlation function has been used for stochastic models of dispersion (Pope, 1994; Sawford, 1991) and for LES of flows over wall mounted cubes (Hanna et al., 2002). More recently, Mordant et al.(2001) carried out an experiment to measure the fully developed turbulent flow in the gap between two counter-rotating disks with $R_{\lambda}=740$, and also confirmed that correlation functions have a form closer to exponential than Gaussian. Typically

$$
R(r, 0,0)=\exp \left(-\frac{\pi r}{2 L}\right),
$$

where $L$ is the length scale. On the basis that correlation functions in most turbulent shear flows have a similar form, we take Eq. (4) as an appropriate model. Of course, the behaviour of the correlation near $r=0$ is not correct, yielding a velocity spectrum which decays at large $1 / r$ like $(1 / r)^{-2}$ rather than $(1 / r)^{-5 / 3}$, but the large-scale behaviour is realistically modelled. We therefore use the exponential correlation function for designing a filter to process a set of random data; for simplicity we start with a set of one dimensional data. With $L=n \Delta x$, where $\Delta x$ is the grid size, the correlation function can be rewritten as

$$
\frac{\overline{u_{m} u_{m+k}}}{\overline{u_{m} u_{m}}}=R_{u u}(k \Delta x)=\exp \left(-\frac{\pi|k|}{2 n}\right) .
$$

A filter function is written as follows,

$$
u_{m}=\sum_{j=-N}^{N} b_{j} r_{m+j},
$$

where $r_{m}$ is a series of random data with $\bar{r}_{m}=0, \bar{r}_{m} r_{m}=1$. The $b_{j}$ are the filter coefficients and $N$ is related to the length scale of the filter. Here we take $N \geq 2 n$. The mean value follows as $\bar{u}_{m}=0$. Because $\overline{r_{m} r_{n}}=0$ for $m \neq n$, we also obtain,

$$
\overline{u_{m} u_{m+k}}=\sum_{j=-N+k}^{N} b_{j} b_{j-k} .
$$


For an arbitrary correlation function $\overline{u_{m} u_{m+k}}$, it is not so straightforward to solve Eq.(7) even numerically (di Mare et al., 2006). However, for a Gaussian (Klein et al., 2003) or an exponential function, it is possible to obtain a very simple but approximate solution.

The filter coefficient $b_{k}$ is first guessed in a form

$$
b_{k}=\exp \left(-\frac{\xi \pi|k|}{n}\right) / \zeta,
$$

where $\zeta$ is simply a normalizing factor to ensure $\overline{u_{m} u_{m}}=1$. The $b_{k}$ is then easily re-written in a form to satisfy $\overline{u_{m} u_{m}}=1$,

$$
b_{k}=\tilde{b}_{k} /\left(\sum_{j=-N}^{N} \tilde{b}_{j}^{2}\right)^{1 / 2}
$$

where

$$
\tilde{b}_{k}=\exp \left(-\frac{\xi \pi|k|}{n}\right) .
$$

It order to obtain a suitable $\xi$, the following expression is to be minimised,

$$
\sigma\left[\sum_{j=-N+k}^{N} b_{j} b_{j-k} /\left(\sum_{j=-N}^{N} \tilde{b}_{j}^{2}\right)-\exp \left(-\frac{\pi|k|}{2 n}\right)\right] \text { for } N \geq 2 n \text { and } n=2, \ldots, 200,
$$

where $\sigma$ is the standard deviation of the square bracket's contents. It was found that $\xi \sim 1.14$ produced a minimum $\sigma$, which is no more than 0.068 for $n=3, \ldots, 200$; whereas $\sigma$ $=0.093$ for $n=2$. The largest individual deviations between the left and right hand terms in expression 11, for $n=2, \ldots, 200$, are less than 0.117 ; the largest occurs near $k=0$ (i.e. near $r=0$ in Eq. 4). For simplicity $\xi$ was chosen as 1, which produced little difference from $\xi=1.14$.

Then the final solution of (7) is,

$$
b_{k}=\tilde{b}_{k} /\left(\sum_{j=-N}^{N} \tilde{b}_{j}^{2}\right)^{1 / 2}, \text { where } \tilde{b}_{k} \simeq \exp \left(-\frac{\pi|k|}{n}\right) .
$$

Again note that (12) is only approximatively valid, in particular for the correlation near $k=0$, but the large-scale behaviour is realistically modelled. Using (6) and (12), a series of (one-dimensional) data with mean value $\bar{u}_{m}=0$, variance $\overline{u_{m} u_{m}}=1$ and prescribed length scale $L$, are obtained. A two-dimensional filter can be obtained as

$$
b_{j k}=b_{j} b_{k},
$$

and this is applied to filter a two-dimensional slice of random data of dimensions $\left[-N_{y}+1\right.$ : $\left.M_{y}+N_{y},-N_{z}+1: M_{z}+N_{z}\right]$, where $M_{y} \times M_{z}$ are the dimensions of the grid in the 
inflow plane, $N_{\alpha} \geq 2 n_{\alpha}, \alpha=y, z$. At the first time step, a two-dimensional slice of data $\Psi_{\beta}(t, y, z), \beta=1,2,3$ with prescribed length scale $L_{\alpha}=n_{\alpha} \Delta \alpha$ is thus obtained.

In the above, it is assumed that constant length scales (in lateral and vertical directions) are used as input at the inlet. To improve the accuracy it would be straightforward to separate the inlet into zones, each having different constant length scales. The twodimensional filter coefficients in (13) must be calculated for each zone. Note that at each time step, the two-dimensional slice of random data is generated only once for all the zones for one velocity component.

For the next time step,

$$
\begin{aligned}
\Psi_{\beta}(t+\Delta t, y, z)= & \Psi_{\beta}(t, y, z) \exp \left(-\frac{\pi \Delta t}{2 T}\right) \\
& +\psi_{\beta}(t, y, z)\left[1-\exp \left(-\frac{\pi \Delta t}{T}\right)\right]^{0.5},
\end{aligned}
$$

where $\psi_{\beta}(t, y, z)$ is obtained in the same way as $\Psi_{\beta}(t, y, z)$ but using a new set of random data. $T$ is the Lagrangian time scale, which can be calculated from measurements or previously computed data. Note $\psi_{\beta}(t, y, z)$ is fully random in time and its variance is unity. Because $\psi_{\beta}(t, y, z)$ is independent of $\Psi_{\beta}(t, y, z)$, it follows from (14) that the variance of $\Psi_{\beta}$ is unity. We obtain the time correlation between $t+k \Delta t$ and $t$ from Eq. (14). Here $\Psi_{\beta}(t, y, z), \Psi_{\beta}(t+k \Delta t, y, z), \psi_{\beta}(t, y, z)$ and $\psi_{\beta}(t+k \Delta t, y, z)$ are written as $\Psi_{\beta, 0}, \Psi_{\beta, k}, \psi_{\beta, 0}$ and $\psi_{\beta, k}$ for simplicity. Again recall that $\psi_{\beta, j}$ is independent of $\Psi_{\beta, 0}$, where $j=0,1,2, \ldots, k$. Using overbars to denote time averages we have that,

$$
\begin{array}{rlrl}
\overline{\Psi_{\beta, 0} \Psi_{\beta, k}} & =\overline{\Psi_{\beta, 0}\left\{\Psi_{\beta, k-1} \exp \left(-\frac{\pi \Delta t}{2 T}\right)+\psi_{\beta, k-1}\left[1-\exp \left(-\frac{\pi \Delta t}{T}\right)\right]^{0.5}\right\}} \\
& =\overline{\Psi_{\beta, 0} \Psi_{\beta, k-1}} \exp \left(-\frac{\pi \Delta t}{2 T}\right), & j=1 \\
& \cdots \\
& =\overline{\Psi_{\beta, 0} \Psi_{\beta, k-j}} \exp \left(-\frac{j \pi \Delta t}{2 T}\right), & j=1 \rightarrow k \\
& \cdots & & \\
& =\exp \left(-\frac{k \pi \Delta t}{2 T}\right), & j=k .
\end{array}
$$

Eq. (15) confirms that Eq. (14) satisfies a time correlation function of exponential form. Also we obtain the space correlation between $\left(y_{1}, z_{1}\right)$ and $\left(y_{2}, z_{2}\right)$ from Eq. (14). Here $\Psi_{\beta}\left(t-j \Delta t, y_{1}, z_{1}\right), \Psi_{\beta}\left(t-j \Delta t, y_{2}, z_{2}\right), \psi_{\beta}\left(t-j \Delta t, y_{1}, z_{1}\right)$ and $\psi_{\beta}\left(t-j \Delta t, y_{2}, z_{2}\right)$ are written as $\Psi_{\beta,-j}^{\prime}, \Psi_{\beta,-j}^{\prime \prime}, \psi_{\beta,-j}^{\prime}$ and $\psi_{\beta,-j}^{\prime \prime}$ for simplicity, where $j=0,1,2 \ldots \infty$.

$$
\begin{aligned}
\overline{\Psi_{\beta, 0}^{\prime} \Psi_{\beta, 0}^{\prime \prime}} & =\overline{\Psi_{\beta,-1}^{\prime} \Psi_{\beta,-1}^{\prime \prime}} \exp \left(-\frac{\pi \Delta t}{T}\right)+\overline{\psi_{\beta,-1}^{\prime} \psi_{\beta,-1}^{\prime \prime}}\left[1-\exp \left(-\frac{\pi \Delta t}{T}\right)\right] \\
& \cdots \overline{\Psi_{\beta,-j}^{\prime} \Psi_{\beta,-j}^{\prime \prime}} \exp \left(-\frac{j \pi \Delta t}{T}\right)+\overline{\psi_{\beta,-j}^{\prime} \psi_{\beta,-j}^{\prime \prime}}\left[1-\exp \left(-\frac{j \pi \Delta t}{T}\right)\right] \\
& \cdots \overline{\psi_{\beta,-\infty}^{\prime} \psi_{\beta,-\infty}^{\prime \prime}}, \quad j=\infty .
\end{aligned}
$$

Recall that $\psi_{\beta}(t, y, z)$ is directly obtained by filtering a two-dimensional slice of random data, which satisfies a space correlation function of exponential form. Hence Eq. (16) 
confirms that the 'interactively' calculated $\Psi_{\beta}$ on the left hand side of Eq. (14) also satisfies a space correlation function of exponential form.

Note that in Eq. (14) $[1-\exp (-\pi \Delta t / T)]^{0.5}$ ranges from $R\left(1.2 L_{x}, 0,0\right)$ to $R\left(2 L_{x}, 0,0\right)$ (see (4)) for $1 / 1000 \leq \pi \Delta t /(2 T) \leq 1 / 100$, where $L_{x}$ is the length scale in $x$ direction. Hence, the contribution of the new slice of random data to the inflow data at the next time step (the second term on the right hand side of (14)) is to some extent equivalent to the contribution of a completely new slice of random data at the next time step, as in Klein et al.'s (2003) original procedure. Overall, (14) is almost equivalent to the full three-dimensional digital filter procedure but we emphasize that (14) only calculates two two-dimensional slices of data, whereas Klein's method calculates $2 N_{x}$ two-dimensional slices. The present method is therefore much more economical, in particular when the longitudinal length scale is large, e.g. typically three times the transverse scales for flows over cubes.

In the above, it is assumed that the Lagrangian time scale $T$ in Eq. (14) is a constant as input for the whole inlet. Recall that the inlet could be separated into zones to provide a variation of the lateral and vertical length scales. Again it is straightforward to allow the time scale $T$ in one zone to differ from the other. Eqs. (15) and (16) are also satisfied for non-constant length scales and time scales.

A comparison of the efficiency of the present inflow generator with Klein's method was carried out for the case 'flow over cubes' discussed in $\S 5$. The three dimensional filter size for Klein's method was $\left(2 N_{x}+1\right) \times\left(2 N_{y}+1\right) \times\left(2 N_{z}+1\right)=97 \times 33 \times 33$. The two dimensional filter size for the current method was $\left(2 N_{y}+1\right) \times\left(2 N_{z}+1\right)=33 \times 33$. The CPU time consumed for the latter was approximately $1 / 500$ times that of the former. As noted in $\S 1$ Hanna et al.(2002) used a method similar to (14) to generate a one-dimensional time series of inflow data, but without spatial correlation in the vertical and lateral directions which, not surprisingly perhaps, is a serious drawback.

Finally, the transformation originally proposed by Lund et al.(1998) is simplified and performed as follows:

$$
u_{i}=\bar{u}_{i}+a_{i j} \Psi_{j},
$$

where

$$
\left[a_{i j}\right]=\left[\begin{array}{ccc}
\left(\hat{R}_{11}\right)^{1 / 2} & 0 & 0 \\
\hat{R}_{21} / a_{11} & \left(\hat{R}_{22}-\left(a_{21}\right)^{2}\right)^{1 / 2} & 0 \\
\hat{R}_{31} / a_{11} & \left(\hat{R}_{32}-a_{21} a_{31}\right) / a_{22} & \left(\hat{R}_{33}-a_{31}^{2}-a_{32}^{2}\right)^{1 / 2}
\end{array}\right]
$$

and $\hat{R}_{i j}$ is the prescribed Reynolds-stress-tensor, estimated from experimental data or a previous numerical simulation with periodic inlet-outlet condition. Note for a boundary layer flow which is homogeneous in the lateral direction, i.e. $z, \hat{R}_{31}=\hat{R}_{31}=0$ and $a_{31}$, $a_{32}$ vanish.

Auto-correlations and two-point correlations of the velocities produced by the inflow generator are shown in Fig. 3 (see more in $\S 4$ ). It is clear that the combination of Eqs. 12, 13 and 14 produces almost exponential correlations. 


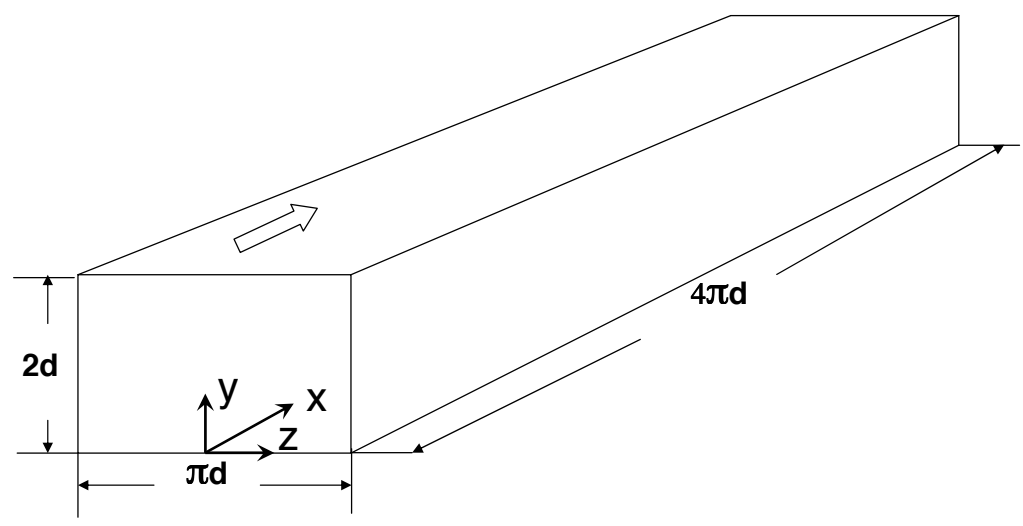

Figure 1. Schematic view of a channel used for inflow boundary condition.

Here, it is assumed that a uniform mesh is used at the inlet. Nevertheless, Eq. (17) can also be applied for a non-uniform mesh at the inlet. One simple method is, firstly to discretise a duplicate of the inlet into a uniform mesh; secondly to perform the digital filtering process and to obtain the three velocity components as in Eq. (17) on the uniform mesh; thirdly to interpolate the velocity components of the uniform mesh onto the nonuniform mesh at the inlet for the computational domain. It is desirable that the difference of the size of the grids between the two sets of meshes is not too large, to minimize the error induced by the interpolation.

\section{Plane channel flows using the new inflow condition}

In order to validate the above digital-filter-based procedure, two test cases were simulated using LES with the inflow boundary condition technique described above - denoted hereafter by IBC. The first was a plane channel flow and is presented in this section. The second was flow over a group of staggered cubes mounted on a wall, presented in the next section.

The computation domain size for the channel flow was $4 \pi d \times 2 d \times \pi d$ where $d$ is the half depth of the channel (see Fig. 1). The axis system in this section is the common 'engineering' one having the $y$ coordinate normal to the wall. The Reynolds number based on the friction velocity and the half depth of the channel $d$ was 180 as in Kim et al.(1987). A mesh of $60 \times 60 \times 50$ was used; it was stretched in the $y$ direction from the walls to the core and had $\Delta_{x}^{+} \approx 38,2 \leq \Delta_{y}^{+} \leq 13, \Delta_{z}^{+} \approx 11$. A duplicate of the inlet was discretised into a uniform mesh $240(y) \times 50(z)$ with a grid space 1.5 wall unit in the $y$ direction. The digital-filtering was performed based on a uniform mesh and the final velocities $(u, v, w)$ were interpolated onto the non-uniform mesh at the inlet of the computational domain. In the core of the channel, the smallest eddies (i.e. having scales less than $\Delta_{y}^{+}=13$ ) generated 

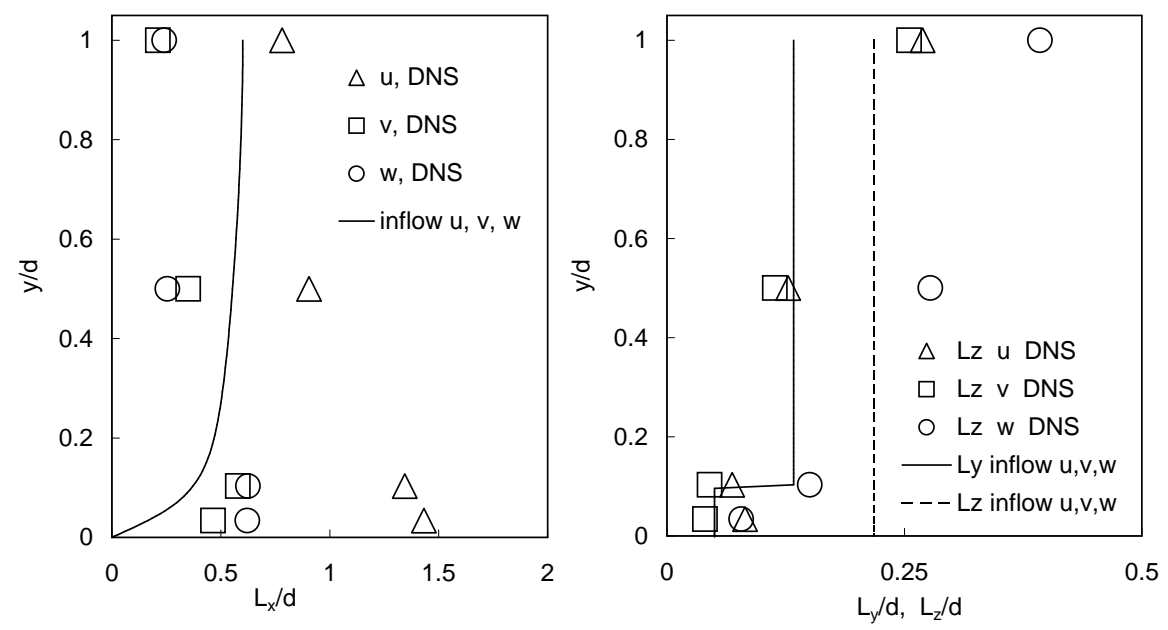

Figure 2. Integral length scales at inlet of plane channel. Symbols, from two-points correlation DNS data (Iwamoto, 2002); lines, for inflow generation.

by the digital-filtering were smeared due to the interpolation. However, the contribution of the small eddies to the turbulent kinetic energy (TKE) was less important than in the near wall region.

The time step $\Delta t$ satisfied the Courant number condition CFL $<1$ and the averaging duration was approximately 61 non-dimensional time units $\left(d / u_{\tau}\right)$ after a fully developed state had been reached. Second-order accurate schemes in both space and time were employed, as described in XC. The synthetic inflow data was imposed at the inlet and zero-gradient outflow conditions at outlet. Periodic boundary conditions were used in the lateral directions. Solid wall boundary conditions were applied for the other boundaries.

First and second velocity moments and integral length scales for the inflow generation were obtained from a plane channel case simulated using full DNS by Kasagi's group and available in a database (No. CH12_PG.WL7 at http://www.thtlab.t.u-tokyo.ac.jp/). For their simulation the domain size was $5 \pi d \times 2 d \times 2 \pi d$ with periodic boundary conditions in both streamwise and spanwise directions. The Reynolds number based on the friction velocity and the half-depth of the channel was 150. A detailed description of the database can be found in Iwamoto (2002).

An LES for the plane channel flow with periodic inlet-outlet conditions was performed (hereafter PBC), for comparison with the results obtained using the inflow technique (IBC). In this case the flow was driven by a pressure gradient $\mathrm{d}\langle P\rangle / \mathrm{d} x_{1}=-\rho u_{\tau}^{2} / d$ (see (1)) which had the same value as in Iwamoto (2002). The domain size, mesh and all other boundary conditions and SGS parameters were the same as for the IBC case. 

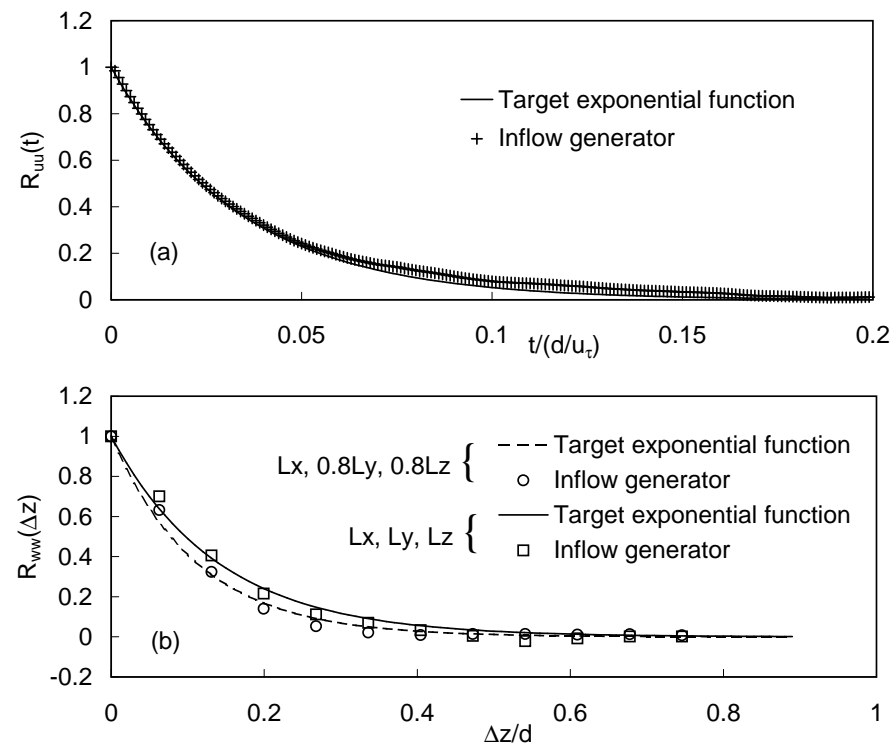

Figure 3. Correlation functions obtained from inflow generator and the corresponding target exponential functions.

By using the following equation,

$$
L_{i}=\int_{0}^{\infty} R\left(x_{i}\right) \mathrm{d} x_{i}, i=1,2,3,
$$

where $R\left(x_{i}\right)$ and $x_{i}$ are respectively the two-point correlation function and the interval in the $i$ th direction, the integral length scales $L_{1}, L_{2}, L_{3}$ (i.e. $L_{x}, L_{y}, L_{z}$ ) were estimated from the two-point correlation data in the database (Iwamoto, 2002).

We emphasise that the aim here was not to generate a particularly accurate simulation of turbulent, smooth-wall channel flow. Rather, our intention is to assess the adequacy of the inflow generation technique for the simulation of up to second order statistics, in the context of wall-bounded flows where there are large variations in length scales. So imposition of precise (e.g. as in (Veloudis et al., 2007)) length scales at the inlet was not attempted. Indeed, given that in a practical urban flow case such length scales can never be more than crudely estimated, it is an advantage for the present purpose not to try to impose precise scales. The heavily simplified forms of the length scale profiles plotted in Fig. 2 were therefore used; these approximate the DNS data only roughly. Because no two-point correlation with wall normal separation was available in the database, $L_{y}$ was simply taken to be $0.67 L_{z}$.

In order to validate the inflow generator, the correlation functions obtained from the inflow generator are compared with the corresponding exponential functions in Fig. 3. Velocity time series at twenty four stations (i.e. $y=1 d, 0 \leq z<1.53 d$ ) produced by the inflow generator were sampled for over ten thousand time steps. Auto-correlations $R_{u u}(t)$, 

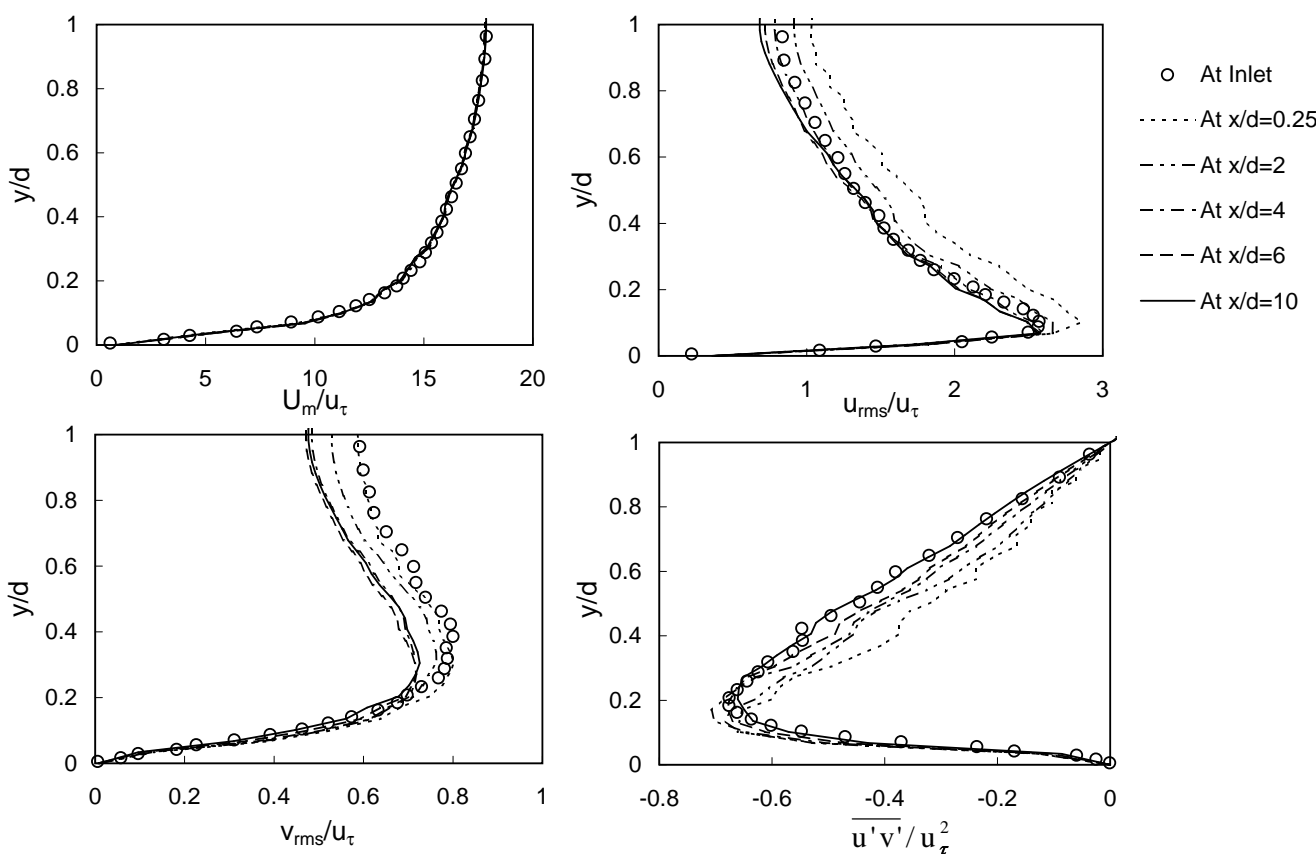

Figure 4. Convergence to fully-developed condition of plane channel flows. Symbols denote values extracted from the DNS database (Iwamoto, 2002).

obtained by post-processing the recorded $u$ time series, are plotted in Fig. 3a. Two-point correlations $R_{w w}(\Delta z)$, obtained by post-processing the recorded $w$ time series, are plotted in Fig. 3b. Two-point correlations $R_{w w}(\Delta z)$ having smaller integral length scales are also shown in Fig. $3 \mathrm{~b}$ - with $L_{x}$ identical to that shown in Fig. 2 and with $L_{y}, L_{z}$ equal to the profiles in Fig. 2 factored by 0.8. The auto-correlations and the two-point correlations of the other velocity components or those in the $y$ direction have the same behaviour as in Fig.3. These confirm that the auto-correlation and two-point correlation functions produced by the inflow generator are closely exponential.

It is crucial to check the convergence of the development in the channel with the artificially generated inflow turbulence. Fig. 4 shows profiles of the normalized streamwise mean velocity, r.m.s turbulence fluctuations and Reynolds shear stress at six typical streamwise stations. The difference between the $U_{m}$ profiles is hard to discern. It is not surprising that there is an evident discrepancy in $u_{r m s}$ and $v_{r m s}$ between $x / d=0.25$ and later locations. The zigzags on the $x / d=0.25$ profile are due to a coarse resolution in the core region. The $w_{r m s}$ profiles (not shown) have a similar trend. Nonetheless, convergence to the fully-developed state is clearly reached by approximately $x / d=10$.

Fig. 5 shows the correlation functions $R_{u u}$ and $R_{w w}$ for the IBC case at various downstream stations at the core of the channel, compared with those for the PBC case. From the inlet to $x / d=6$, the correlation functions are similar, and close to a decaying exponential. It was also noted that the vertical profiles of the integral time scales at $x / d=10, z=0$ for the IBC case are comparable with those for the $\mathrm{PBC}$ case. It is 

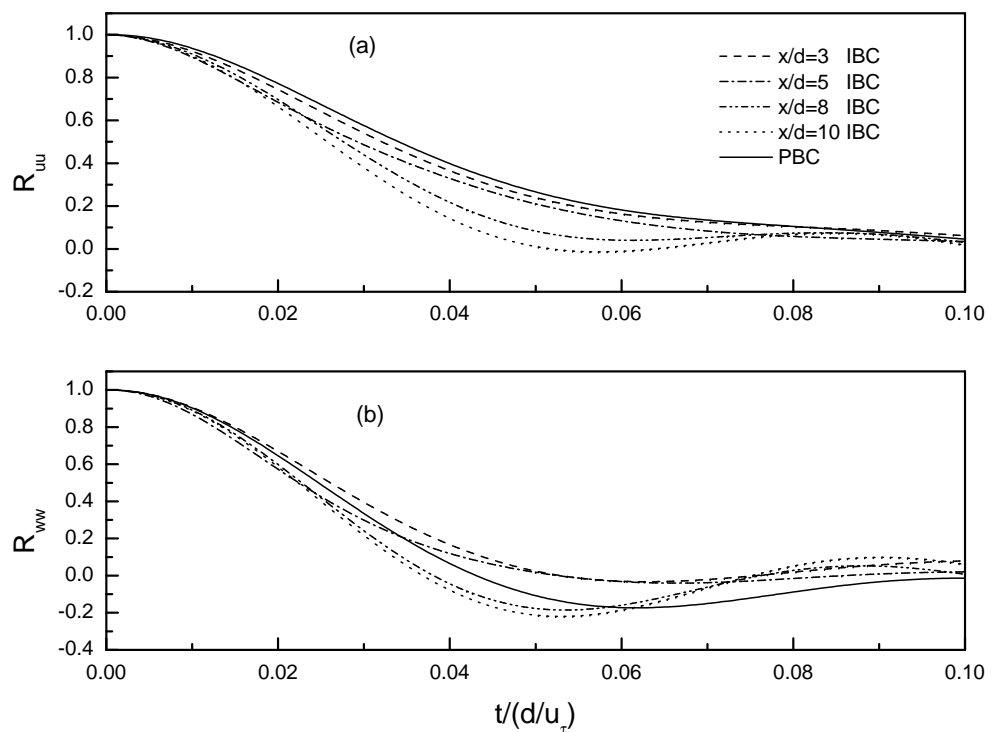

Figure 5. Correlation functions $R_{u u}$ (a) and $R_{w w}$ (b) at $x / d=3, x / d=5, x / d=8, x / d=10(y=d$, $z=0$ ) for inflow BC case, and averaged $R_{u u}$ (a) and $R_{w w}$ (b) over the central line $(y=d, z=0)$ for periodic BC case.

interesting to note that the heavily simplified inflow conditions (as in Fig. 2) produce integral length scales at $x / d=6$ in reasonable agreement with those for the $\mathrm{PBC}$ case, which suggests that imposition of precise length scales at the inlet are not crucial to the LES computations. There is no doubt that an improvement of the imposed length scales at the inlet reduces the discrepancy of the correlation functions between the IBC and PBC cases at large time scales. Note that from $x / d=8$ the turbulence length scale slightly decreases, both for $R_{u u}$ and $R_{w w}$, which is probably due to the zero gradient outlet boundary conditions - for $x / d=10$ this is applied at $t u_{\tau} / d \approx 0.15$.

The sensitivity of the downstream statistics to the integral length scales imposed at the inlet was carefully investigated. The same inflow turbulence profiles obtained from Iwamoto (2002) were used in every case. But various different length scale combinations were used $-L_{x}$ identical to that shown in Fig. 2 and with $L_{y}, L_{z}$ equal to the profiles in Fig. 2 factored by $1.3,1,0.9$ or 0.8 . Fig. 6 plots the mean velocity, velocity r.m.s. and Reynolds shear stress at $x / d=10$. Note first that these variations in prescribed length scales had little influence on the mean streamwise velocity, but the influence on the velocity r.m.s. and the Reynolds shear stress is visible. A $50 \%$ variation in $L_{y}$ and $L_{z}$ generated a variation less than $10 \%$ in $v_{r m s}$ and less than $13 \%$ in $\overline{u^{\prime} v^{\prime}}$. In general, the second moments increase slightly with the increasing of prescribed length scales in the range shown in Fig.6.

Fig.7 shows the evolution of the local friction velocity $\hat{u}_{\tau}(x)$, which was obtained by averaging in the lateral direction. For all the four cases, the variation of the local skin friction downstream from $x=10 d$ is less than $0.2 \%$, so we assume that the local skin friction converges at $x=10 \mathrm{~d}$. It is not surprising that the convergence over such a short domain is slightly dependent on the length scales imposed at the inlet. However, a 50\% 

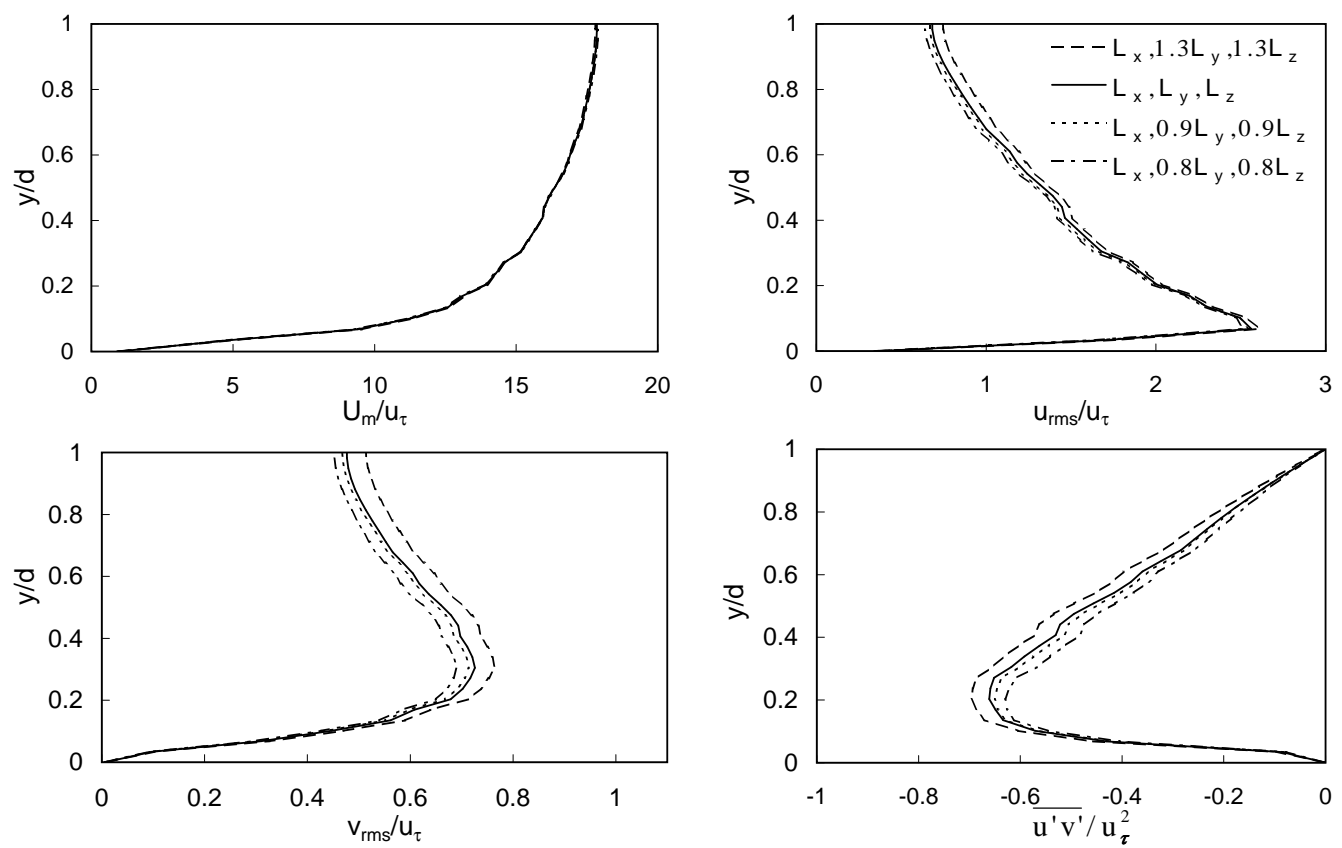

Figure 6. Influence of the integral length scales on mean velocity, velocity r.m.s. and Reynolds shear stress at $x / d=10$.

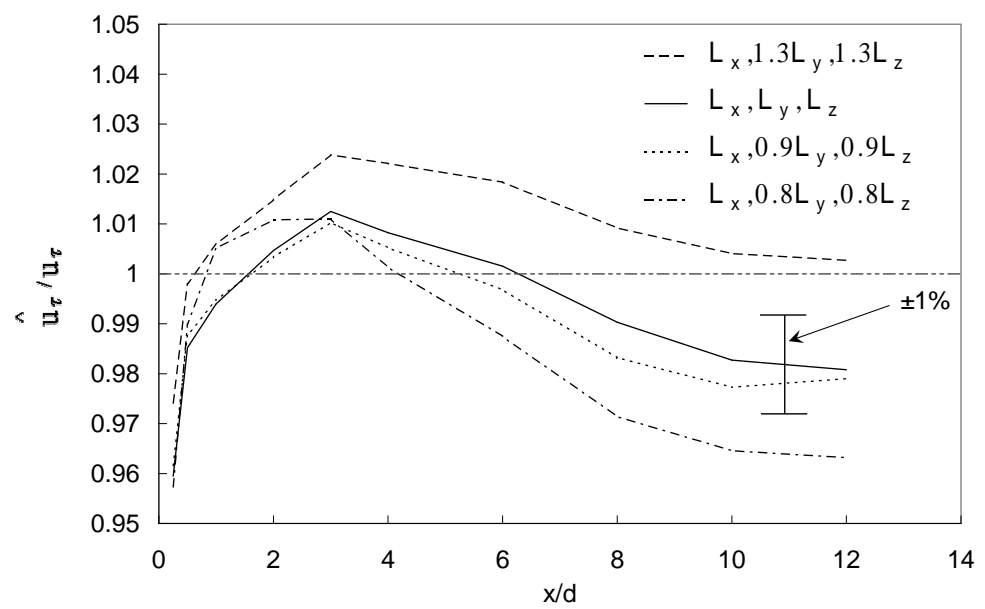

Figure 7. Evolution of the local friction velocity $\hat{u}_{\tau}(x)$. 

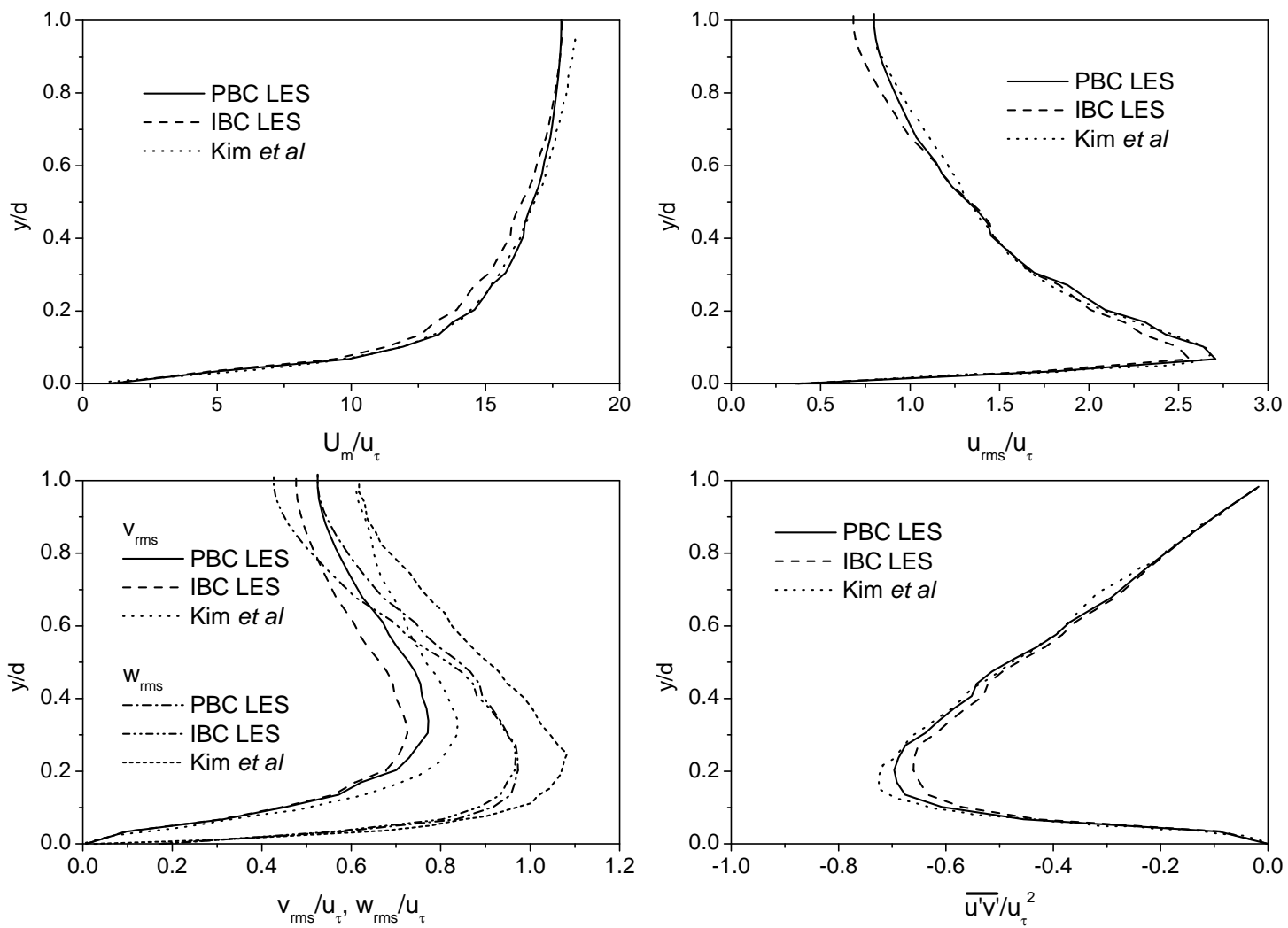

Figure 8. Comparison of mean velocity, velocity r.m.s. and Reynolds shear stress between with inflow boundary conditions and with periodic boundary conditions.

variation in $L_{y}$ and $L_{z}$ generated a variation less than $4 \%$ in $\hat{u}_{\tau}$ at $x=10 d$. Overall, we conclude that the sensitivity to the inlet length scales on the statistics at $x=10 d$ is not too large for these channel flows.

Fig. 8 shows vertical profiles of the turbulence statistics at $x / d=10$ obtained from the inflow BC case compared with those from the periodic BC case as well as the Kim et al. (1987) DNS results. Overall, the comparison between the two cases is very satisfactory. With a set of more carefully estimated length scales imposed at the inlet, it is likely that the two sets of profiles would be in even better agreement with each other. Note, finally, that the main reason for the differences in $v_{r m s}$ and $w_{r m s}$ between the periodic BC case and the DNS calculations is the relatively low resolution of the LES and, perhaps, the inadequacy for this smooth-wall case of the wall boundary condition and/or the sub-grid-scale model.

Note that $x / d=10$ is a short distance for development to a fully turbulent flow. Recall that, as noted earlier, Keating et al. (2004) used Batten et al. (2004)'s procedure (i.e. a synthetic turbulence generation method) to generate inflow data for a plane channel flow and found that the redevelopment was slow requiring at least $20 \mathrm{~d}$. For instance, at 


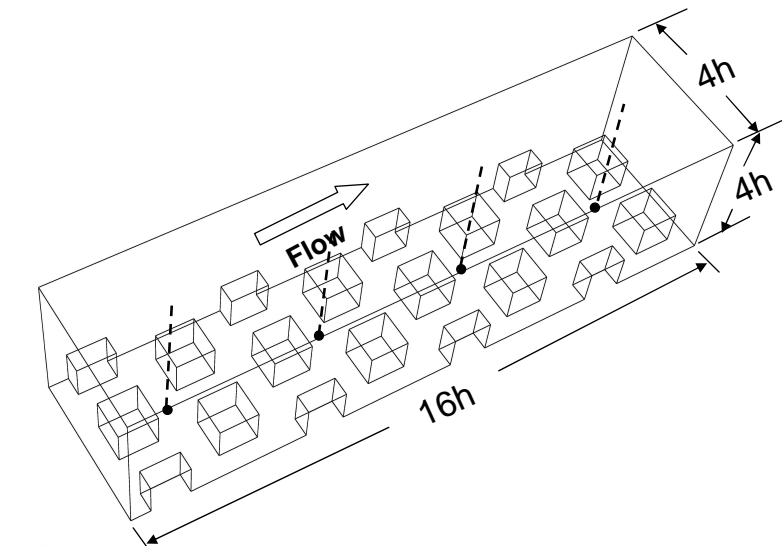

$\int_{y}^{2} x$ (a)

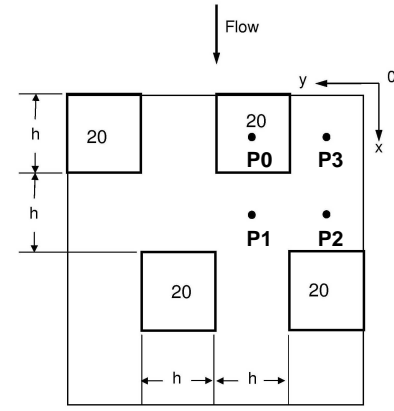

(b)

Figure 9. (a) Schematic view of the domain used for the inflow BC computations. (b) Plan view of the domain for periodic $\mathrm{BC}$ computations.

$x=10 d$ the coefficient of friction produced by Batten et al.'s procedure reaches less than $70 \%$ of that produced by a 'precursor simulation' inflow generator. We are not yet quite sure why different synthetic inflow generators yield such different outputs. But we are sure that digital-filter based inflow generators are promising procedures, and our proposed technique is very efficient and has an accuracy similar to earlier digital filter methods.

\section{Flow over a staggered cube array using the new inflow condition}

The details of the parameters used for these calculations can be found in our previous paper (XC), in which LES with a periodic inlet-outlet condition was applied to calculate the turbulent flow over staggered wall-mounted cubes.

Here only a brief description of the computational domain and the boundary conditions are given. Fig. 9a is a schematic view of the domain used for the inflow BC case; it has eight rows of staggered cubes (four of the repeated units of the periodic case stacked in the streamwise direction). Note that the view in Fig. $9 \mathrm{~b}$ is shifted $0.5 \mathrm{~h}$ in the lateral direction from that of Fig. 9a. Also note that the $z$ coordinate is normal to the wall in these cases, consistent with XC and the corresponding wind tunnel experiments (Castro et al., 2006). The four vertical lines indicate data sampling locations and will subsequently be denoted (from left to right) by 'behind row 1', 'behind row 3', 'behind row 5' and 'behind row 7'. These 4 stations correspond to the 'P1' station in the periodic BC case (Fig. 9b). Fig.9b shows a plan view of one unit of the staggered wall-mounted cube array, which was also the computational domain for the periodic BC case in both inlet-outlet and lateral directions (XC). The domain height is $H=4 h$, where $h$ is defined as the cube height. The plan area 


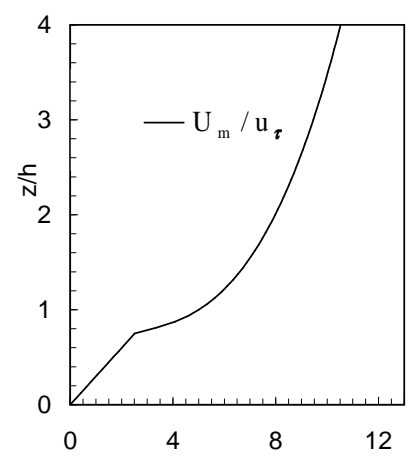

(a), mean velocity

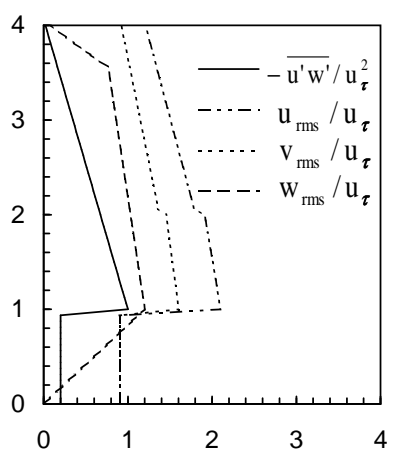

(b), second moments

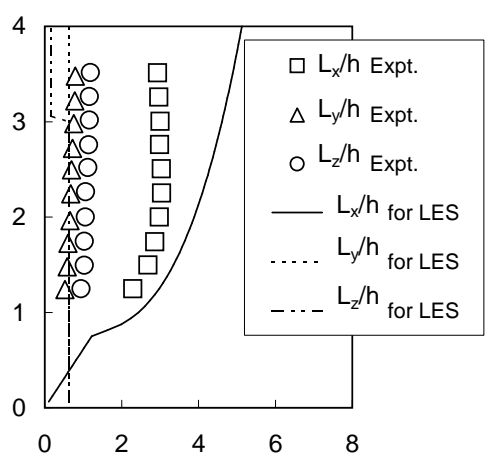

(c), integral length scales

Figure 10. Turbulence statistics (a,b) and integral length scales(c) at inlet.

density of the cubic array was 0.25 . P0, P1, P2 and P3 indicate four typical data sampling locations.

The synthetic inflow data was imposed at the inlet and zero-gradient outflow conditions at outlet. At the top of the domain, stress free conditions were applied. Periodic boundary conditions were used in the lateral directions. Solid wall boundary conditions with a wall model were applied for all other boundaries (see details in XC). A uniform mesh of more than one million cells with $16 \times 16 \times 16$ grid points per cube was used, which was suggested by $\mathrm{XC}$ for sufficient accuracy in these kinds of flows.

The turbulence statistics and the integral length scales which were applied for the generation of the inflow data are shown in Fig. 10. Note that the $U_{m}(z), \overline{u^{\prime} w^{\prime}}(z), u_{r m s}(z)$, $v_{r m s}(z), w_{r m s}(z)$ profiles are not functions of $y$ for the current simulations. This could easily be done (as discussed in $§ 3$ ) but in practical cases it is unlikely that sufficient knowledge of such spanwise variations would be available to make it either sensible or possible; recall that one large-scale (UM) grid cell is larger than the entire local domain being computed by the LES and thus only one profile can be obtained from the UM to drive the LES computation, perhaps updated after an appropriately large number of LES timesteps to make use of the long-time scale variations provided by the large-scale model. The turbulence profiles were chosen to approximate the horizontally averaged measurements obtained by (Castro et al., 2006). They are considerably simplified and thus differ from the 'real' values at some stations within the canopy, but the LES results were not found to be very sensitive to these discrepancies. We found that having a lower value of $L_{z}$ in the vicinity of the upper free-slip plane (Fig. 10c) gave better performance. The length scale profiles shown were the default scales always applied unless otherwise stated.

The initial duration of the computations was over $200 T\left(T=h / u_{\tau}\right)$, whereas the subsequent averaging duration for all the statistics was approximately $60 T$. Here for bodyforce driven channel flow, $u_{\tau}$ is the effective friction velocity. As found by $\mathrm{XC}$, the variations 

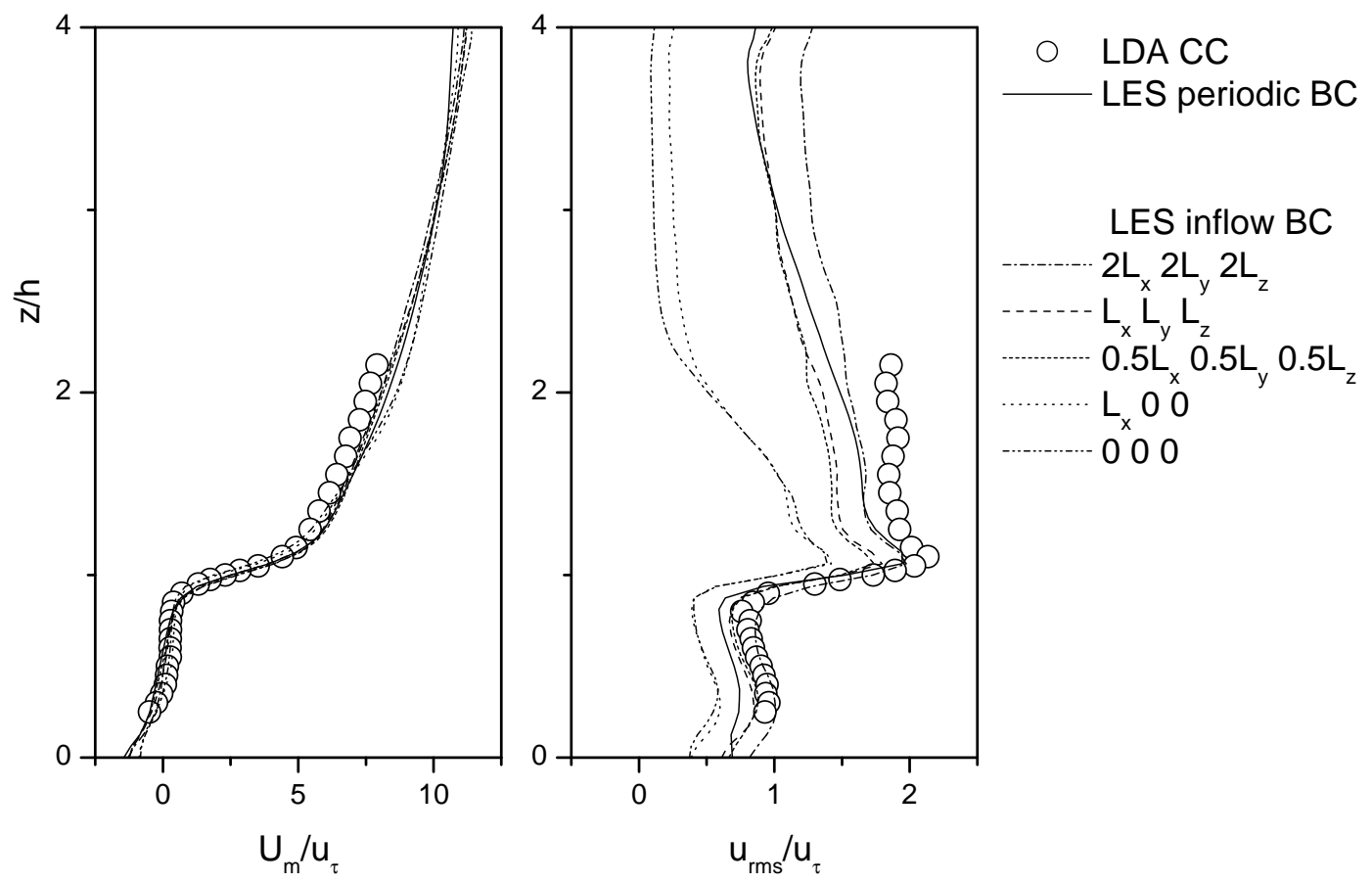

Figure 11. Influence of the integral length on the mean velocity and the turbulence intensities. "LDA CC" and "LES periodic BC", at "P1" as in Fig.9; "LES inflow BC", behind row 7 .

in statistical data throughout the roughness sub-layer (including the canopy region) were usually small once the averaging duration exceeded $20 T$.

Fig. 11 shows the influence of the integral length scales imposed at the inlet. The inflow turbulence profiles in Fig. 10b were used in every case, but various different length scale combinations were used - either identically zero for all three, zero for $L_{y}$ and $L_{z}$ but with the $L_{x}$ shown in Fig. 10c, or all three non-zero and equal to the profiles in Fig. $10 \mathrm{c}$ factored by $0.5,1$ or 2 . Note first that these variations in prescribed length scales had little influence on the mean streamwise velocity, but fully random fluctuations (i.e. zero length scales) at the inlet led to a rapid downstream decay of turbulence, particularly in the region above the canopy $(z / h>2)$. However, within and immediately above the canopy, the five upstream rows of cubes have themselves generated considerable turbulence - quite a different behaviour from what would be expected in the smooth-wall channel flow. So flows over group of cubes, or indeed any collection of large roughness elements, are expected to be much less sensitive to the precise inflow condition than are smooth-wall flows.

With only a temporal correlation at the inlet (i.e. $L_{x}$ the only non-zero length scale) the behaviour improved very little compared with the completely random fluctuations case. This result is not as good as Hanna et al.'s (2002) finding, which may be due to the different upper boundary conditions and the particular integral length $L_{x}$ used at the inlet. 

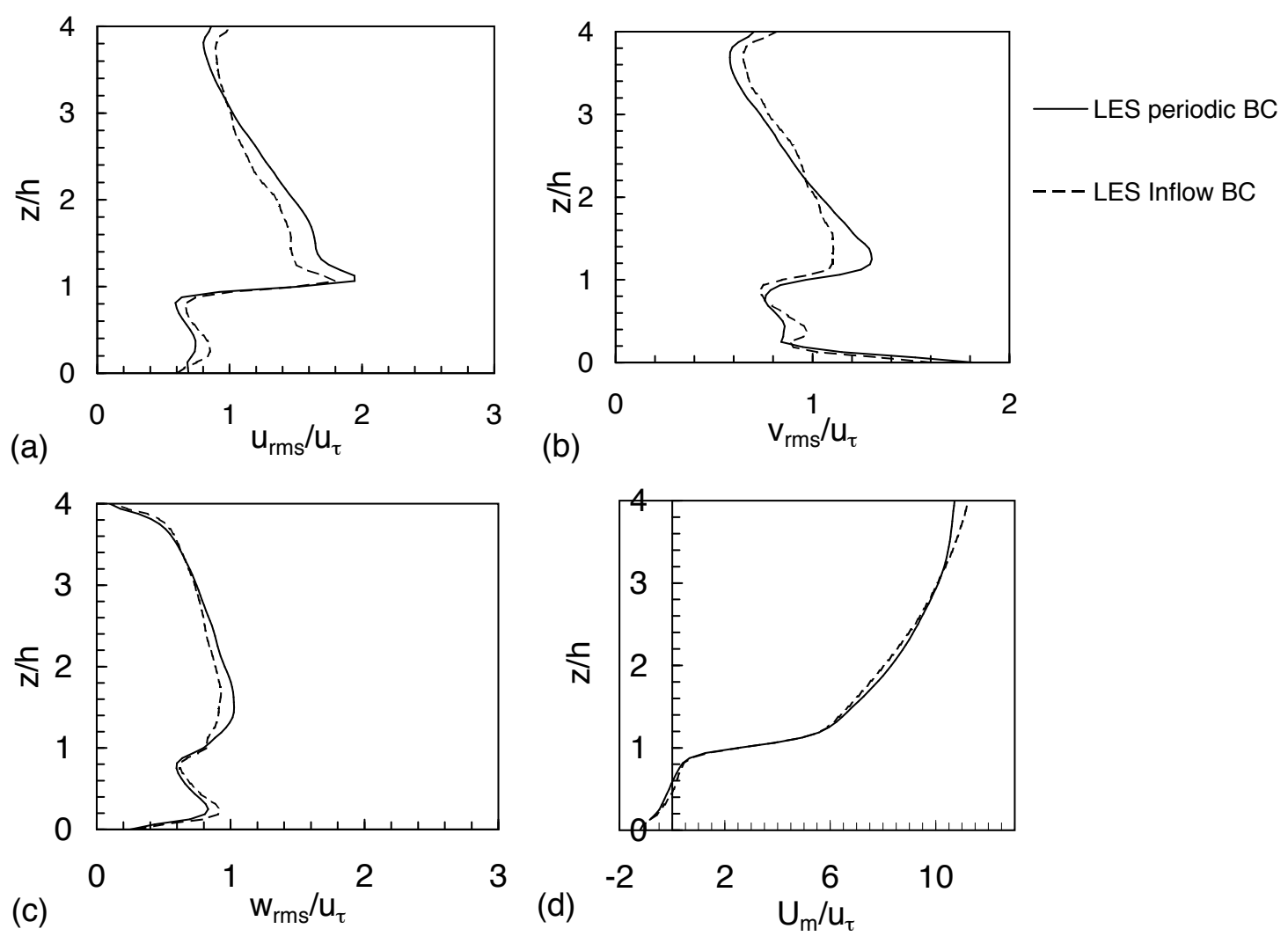

Figure 12. Vertical profiles of turbulence intensities (a, b, c) and mean streamwise velocity (d). 'LES periodic BC", at "P1" as in Fig.9; "LES inflow BC", behind row 7.

However, imposing the scales $0.5 L_{x}, 0.5 L_{y}$ and $0.5 L_{z}$ shows a great improvement over the fully random case and with the full scales shown in Fig. 10c the results are in even better agreement with the measurements and those of the periodic BC case, at least within and immediately above the canopy. The discrepancy between the periodic BC and inflow BC cases above the canopy is most probably due to the integral length scales in this region being too low (see Fig. 10) - doubling the length scales $\left(2 L_{x}, 2 L_{y}, 2 L_{z}\right)$ improves the second moments in the region just above the canopy but leads to an overestimate of the second moments (compared with the DNS data) in the upper part of the flow. Profiles of the other second moments had a similar trend. Overall, Fig. 11 shows that whilst the results far above the canopy are not totally insensitive to the precise inflow length scales, within or immediately above the canopy such sensitivity is, perhaps not surprisingly, relatively small.

Fig. 12 shows a comparison between LES with inflow BC and LES with periodic BC (XC). The length scales for the inflow BC case were those shown in Fig. 10. Discrepancies in the r.m.s. fluctuations within the canopy might be a result of the assumption of homogeneity in the lateral direction at the inlet (see Fig. 10 and §4). Again, the discrepancy 


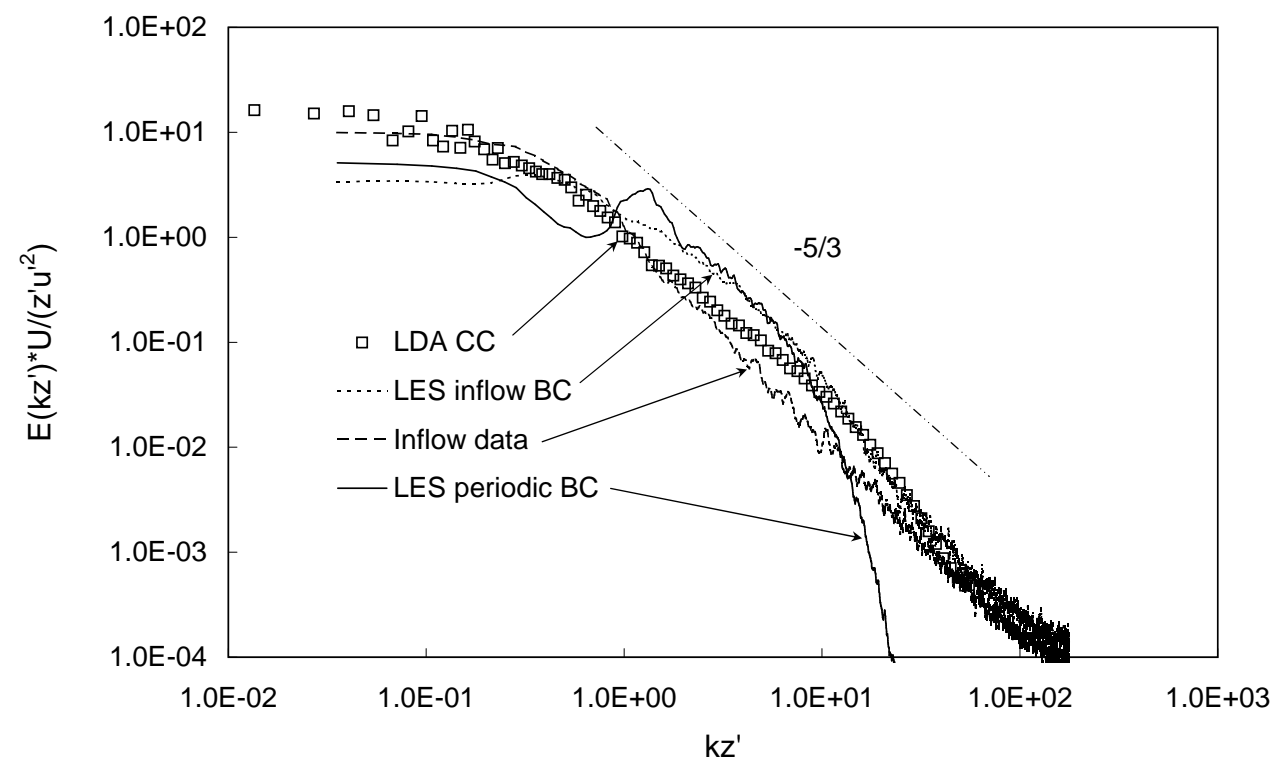

Figure 13. Spectra of the axial turbulence component, plotted in inner-layer scaling. "LES inflow BC" was sampled at $z=1.5 h$ on row seven.

immediately above the canopy is most probably due to the low length scales imposed in this region. Nevertheless, the comparison is promising.

Fig. 13 shows typical spectra, plotted in inner-layer scaling. $k=2 \pi f / U_{m}$ is the wavenumber and $z^{\prime}=z-d$, where $d=0.013 \mathrm{~m}$ is the zero-plane displacement (Castro et al., 2006). The data were sampled at $z=1.5 h$ (on row seven for the inflow BC case). More than one decade of inertial sub-range with slope $-5 / 3$ is seen in the spectrum obtained from measurements at a Reynolds number of approximately 5000 (Meinders \& Hanjalić, 1999; Castro et al., 2006). Over a smooth wall, such a wide inertial sub-range is usually only found at much higher Reynolds number. This again confirms that turbulence generated by urban-like obstacles (with sharp edges) is large-scale dominated. In contrast, the periodic $\mathrm{BC}$ case resolves a relatively narrow inertial sub-range. There is a rapid drop beyond about $k z^{\prime}=10$, which is due to the relatively low spatial resolution. However, the inflow BC case is in better agreement with the measurements, which again suggests that the method is satisfactory. Note in particular that the rapid drop at high frequencies is not present in this case, which must be because the inlet spectrum is more fully resolved in time and the axial fetch is too short for the finite spatial resolution of the LES to "degrade" the spectrum to the form it has when periodic boundary conditions are imposed. Note that the spatial and the temporal resolutions in the IBC computations are the same as those in the PBC computations.

We stress again that the technique shortens the developing distance even more for spatially evolving turbulence flow over a very rough wall (e.g. over an array of cubes) than over a smooth wall. In the simulation of flows over a real urban area, precise geometry 
upstream of the area of interest is usually unknown. We have found it useful for practical cases to set up an array of artificial building blocks in the upstream part of the computational domain. The present inflow technique needs fewer such artificial blocks to achieve development of appropriate turbulence at the leading edge of the area of interest.

\section{Conclusion and further work}

The final objective of the current research is coupling weather-scale computations with street scale large-eddy simulation. It is the efficiency of the street scale computation which provides the major bottle-neck in such coupling. Other issues, such as Reynolds number dependency, the minimum resolution needed and the importance of the wall layers on the block surfaces, were addressed in Xie \& Castro (2006a). The method proposed here for inserting appropriate turbulence at the inlet, based on exponential (rather than Gaussian) velocity correlation functions, is a modified form of the digital filter based method originally proposed by Klein et al.(2003) and recently developed in newer versions (Kempf et al., 2005; di Mare et al., 2006; Veloudis et al., 2007). Note in this method the digital filter approach is used only for the generation of spatially-correlated two-dimensional slices of data, while the two-dimensional slice data at the current time step are correlated with the two-dimensional slice data at the previous time step by using an exponential correlation function. Hence it is much more efficient than the earlier fully three-dimensional digital filter methods.

LES simulations of plane channel flows and flows over a group of staggered cubes have provided satisfactory validation of the technique, with results showing good agreement with simulations using periodic inlet-outlet boundary conditions and reasonable agreement with data from other sources - both the DNS of Kim et al.(1987) and the laboratory experiments of Castro et al.(2006). These satisfactory validations, the fact that the results are not too sensitive to the precise form of the prescribed inlet turbulence, and the high efficiency of the technique, together suggest that the method will be very useful for practical simulations of urban-type flows.

It is worth emphasizing that all digital filter based methods generate artificial turbulence, in which most crucial quantities -length scale, spectra, mean velocity, Reynolds stresses - are approximated more or less satisfactorily. There is certainly scope for further development. For instance, the artificially generated turbulence may not be divergence free and this may have some downstream effects; this issue has rarely been addressed in the literature. A more general question, perhaps, relates to just how detailed should be the imposed statistics and, indeed, how many of the independent quantities need to be considered. Our feeling is that for general applicability it is important to model not only the turbulence stresses but also correlation scales in all three directions, as in the present method. However, only three of the correlation length scales have been explicitly modelled (the others are implicitly assumed equal to these, as appropriate). Modelling all nine separately would be straightforward but the effects would, arguably, be small. 
Our method has also been applied to simulate combined oscillatory through-flow and a steady current over an array of staggered cubes, and was validated by using inlet-outlet periodic boundary conditions driven by an appropriate unsteady pressure gradient. In addition, it has been used to simulate flow and point source dispersion over a real urban geometry - the DAPPLE field site (Marylebone Rd region, London, http://www.dapple.org.uk/); numerical results are in good agreement with wind tunnel measurements and the work will be reported in due course. These last two test cases are not discussed here in the interests of space. We are now developing tools for implementing dynamic spatial boundary conditions derived from the output of much larger-scale computations, like those available from the UK Met Office's Unified Model, coupled with the new small-scale turbulence inflow method described here, to simulate flows over more complex geometry.

Acknowledgments: This project was supported by NCAS, NERC, under Grant No. DST/26/39. Numerical simulations were performed on the IRIDIS computational system, ISS, University of Southampton. The CFD code providers were also very helpful in ensuring appropriate implementations of their codes on the ISS system. We are also grateful to the referees for their helpful comments, which led to improvements in the paper.

\section{References}

Batten P., Goldberg U. and Chakravarthy S., 2004, Interfacing statistical turbulence closures with large-eddy simulation, AIAA J. 42(3) 485-492.

Britter R.E. and Hanna S.R., 2003, Flow and dispersion in urban areas, Ann. Rev. Fluid Mech. 35 469-496.

Castro I.P., Cheng H. and Reynolds R., 2006, Turbulence over urban-type roughness: deductions from wind tunnel measurements, Bound. Layer Meteorol., 118 109-131.

Collier C.G., 2006, The impact of urban areas on weather, Q.J.R. Meteorol. Soc. 132 1-25.

di Mare L., Klein M., Jones W.P. and Janicka J., 2006, Synthetic turbulence inflow conditions for large-eddy simulation, Phys. Fluids 18(2).

Druault P., Lardeau S., Bonnet J.-P., Coiffet F., Delville J., Lamballais E., Largeau J.F. and Perret L., 2004, Generation of three-Dimensional turbulent inlet conditions for large-eddy simulation, AIAA J. $42(3)$ 447-456.

García-Villalba M., Fröhlich J. and Rodi W., 2004, On inflow boundary conditions for large eddy simulation of turbulent swirling jets. In Proc. 21st Int. Congress of Theoretical and Applied Mechanics, Warsaw, Poland, Gutkowski W., Kowalewski T.A. (Eds.), Springer Verlag.

Iwamoto K., 2002, Database for fully developed channel flow, THTLAB Internal Report (ILR0201), Dept. Mech. Eng., Univ. Tokyo. DNS database (CH12_PG.WL7), http://www.thtlab.t.utokyo.ac.jp/.

Jarrin N., Benhamadouche S., Laurence D. and Prosser R., 2006, A synthetic-eddy-method for generating inflow conditions for large eddy simulation, Int. J. Heat Fluid Flow 27(4) 585-593.

Johansson P.S., and Andersson H.I., 2004, Generation of inflow data for inhomogeneous turbulence, Theore. and Comput. Fluid Dynamics 18(5) 371-389.

Hanna S.R., Tehranian S., Carissimo B., Macdonald R.W. \& Lohner R., 2002, Comparisons of model simulations with observations of mean flow and turbulence within simple obstacle arrays, Atmos. Environ. 36 5067-5079. 
Kondo K., Murakami S. and Mochida A., 1997, Generation of velocity fluctuations for inflow boundary conditions of LES, J. Wind Eng. Ind. Aerodyn. 67-68 51C64.

Keating A., Piomelli U., Balaras E. and Kaltenbach H.-J., 2004, A posteriori tests of inflow conditions for large-eddy simulation, Phys. Fluids, 16 (12),4696-4712.

Kempf A., Klein M and Janicka J., 2005, Efficient generation of initial- and inflow-conditions for transient turbulent flows in arbitrary geometries. Flow, Turbulence and Combustion 74 67-84.

Kim J., Moin P. and Moser R., 1987, Turbulence statistics in fully developed channel flow at low Reynolds number, J. Flui Mech. 177 133-166.

Klein M., Sadiki A. and Janicka J., 2003, A digital filter based generation of inflow data for spatially developing direct numerical simulation or large eddy simulation. J. Comp. Phys. 186 652-665.

Le H. and Moin P., 1994, Direct numerical simulation of turbulent flow over a backward-facing step, Tech.Rep. TF-58,Standford University.

Le H., Moin P. and Kim J., 1997, Direct numerical simulation of turbulent flow over a backwardfacing step, J. Fluid Mech. 330 349-374.

Lee S., Lele S. and Moin P., 1992, Simulation of spatially evolving compressible turbulence and the application of taylors hypothesis, Phys. Fluids A 4 1521-1530.

Lund T., Wu X. and Squires D., 1998, Generation of turbulent inflow data for spatially developing boundary layer simulation. J. Comp. Phys. 140 233-258.

Meinders E.R. \& Hanjalić K., 1999, Vortex structure and heat transfer in turbulent flow over a wall-mounted matrix of cubes, Int. J. Heat Fluid Flow 20 255-267.

Mordant N., Metz P., Michel O. and Pinton J.-F., 2001, Measurement of Lagrangian velocity in fully developed turbulence. Phys. Rev. Lett. 87(21).

Perret L., Delville J., Manceau R. and Bonnet J.P., 2006, Generation of turbulent inflow conditions for large eddy simulation from stereoscopic PIV mearurements, Int. J. Heat Fluid Flow 27(4) 576-584.

Pope S.B., 1994, Lagrangian PDF methods for turbulent flows, Annu. Rev. Fluid Mech. 26 23-63.

Sandham N.D., Yao Y.F. and Lawal A.A., 2003, Large-eddy simulation of transonic turbulent flow over a bump, Int. J. Heat Fluid Flow 24(4) 584-595.

Sawford B.L., 1991, Reynolds number effects in Lagrangian stochastic models of turbulent dispersion, Phys. Fluids 3 (6) 1577-1586.

Stoesser T., Mathey F., Frohlich J. and Rodi W., 2003, LES of flow over multiple cubes. ERCOFTAC Bulletin No. 56.

Veloudis I., Yang Z., McGuirK J.J., Page G.J. and Spencer A., 2007, Novel implementation and accessment of a digtial filter based approach for the generation of LES inlet conditions, Flow, Turbulence and Combustion, 79 (1), 1-24.

Xie Z.-T., Castro I.P., 2005, LES for flow over urban-like surfaces, Euromech Colloquium 469, Oct. 2005, Dresden, Germany.

Xie Z.-T., Castro I.P., 2006, LES and RANS for turbulent flow over arrays of wall-mounted cubes, Flow, Turbulence and Combustion, 76 (3), 291-312.

Xie Z.-T., Castro I.P., 2006, Large-eddy simulation for urban micro-meteorologyr, Proceedings of the Conference of Global Chinese Scholars on Hydrodynamics, Jul. 2006, Shanghai, In: $J$. Hydrodynamics, 18(3, Supplement 1), 259-264. 